\title{
Modifications of the Lifshitz-Kosevich formula in two-dimensional Dirac systems
}

\author{
Carolin Küppersbusch ${ }^{1,2}$ and Lars Fritz ${ }^{1}$ \\ ${ }^{1}$ Institute for Theoretical Physics and Center for Extreme Matter and Emergent Phenomena, Utrecht University, \\ Leuvenlaan 4, 3584 CE Utrecht, The Netherlands \\ ${ }^{2}$ Institut für Theoretische Physik, Universität zu Köln, Zülpicher Straße 77, 50937 Köln, Germany \\ (Received 22 November 2016; revised manuscript received 23 May 2017; published 6 November 2017)
}

\begin{abstract}
Starting from the Luttinger-Ward functional [J. M. Luttinger and J. C. Ward, Phys. Rev. 118, 1417 (1960)] we derive an expression for the oscillatory part of the grand potential of a two-dimensional Dirac system in a magnetic field. We perform the computation for the clean and the disordered system, and we study the effect of electron-electron interactions on the oscillations. Unlike in the two-dimensional electron gas (2DEG), a finite temperature and impurity scattering also affects the oscillation frequency. Furthermore, we find that in graphene, compared to the 2DEG, additional interaction induced damping effects occur: to two-loop order electron-electron interactions do lead to an additional damping factor in the amplitude of the Lifshitz-Kosevich formula.
\end{abstract}

DOI: 10.1103/PhysRevB.96.205410

\section{INTRODUCTION}

A major breakthrough in the investigations of electronelectron interactions in many-body systems was the Landau theory of the Fermi liquid [1]. It explains why a system of strongly interacting particles can be described by a system of noninteracting quasiparticles, which allows for simple theoretical models to describe phenomena in condensed-matter systems. However, in Dirac systems, Fermi-liquid theory is not straightforwardly applicable [2]. It has been shown that they can be described by a marginal Fermi liquid [3]: since the screening length diverges at the Dirac point, electron-electron interactions are in principle expected to play a significant role.

One important example of a two-dimensional Dirac system is graphene. In general there exist two different predictions on the effect of Coulomb interactions in graphene. Assuming a weak-coupling perspective, electron-electron interactions are assumed to renormalize the Fermi velocity according to $v_{F} \rightarrow$ $v_{F} \ln \frac{\Lambda}{k}$, where $k$ is the momentum and $\Lambda$ is a high-energy cutoff [4-6]. At strong coupling one expects the system to be an excitonic insulator [7,8]. Different approaches yield different critical interaction strengths; some suggest that graphene in vacuum might be an insulator, while experimental evidence rather points toward it being weakly coupled $[5,10]$.

There are only very few experiments which allow one to deduce information about electron-electron interactions and it would be desirable to have other experimental probes which one can compare to theory predictions. A standard experiment in the determination of electronic properties of conductors is the measurement of quantum oscillations in both transport (Shubnikov-de Haas) and thermodynamic (de Haas-van Alphen) quantities. Generally, the amplitude of the oscillations is described by the Lifshitz-Kosevich (LK) formula $[9,11,12]$. As electron-electron interactions enter the oscillation amplitude, one can extract information about it by fitting the LK formula to the measured amplitude [5,12]. In a two-dimensional electron gas (2DEG) in the Fermi-liquid regime, the amplitude of the LK formula was shown to not contain an additional damping factor due to electron-electron interactions which could be associated with an effective temperature, but instead electron-electron interactions only affect the oscillations by renormalizing the cyclotron frequency or fermionic mass $[13,14]$. It has to be pointed out that a similar statement was made for the electron-phonon problem $[12,15,16]$.

Since a Dirac system near the charge neutrality point is not a true Fermi liquid [2,3], we ask the question whether the LK formula for a Fermi liquid still holds. We also ask this question in view of experiments where the Fermi-liquid LK formula was used to extract interaction effects from the damping of the amplitude of magnetic oscillations in graphene [5], albeit for transport measurements.

\section{A. Model}

Dirac systems are characterized by a linear dispersion resulting from the Hamiltonian,

$$
\mathcal{H}=v_{F} k_{x} \hat{\sigma}_{x}+v_{F} k_{y} \hat{\sigma}_{y}-\mu \mathbb{1},
$$

where $\mathcal{H}$ is the effective low-energy Bloch Hamiltonian, $v_{F}$ is the Fermi velocity, $\mu$ corresponds to the chemical potential, and the Pauli matrices $\hat{\sigma}_{x}$ and $\hat{\sigma}_{y}$ in graphene, for instance, act in sublattice space, while on the surface of a three-dimensional topological insulator they would act in spin space.

The orbital effect of an external magnetic field, $B$, is in this formulation accounted for in the standard form of the minimal coupling according to $\vec{k} \rightarrow \vec{k}-e \vec{A}$, where $\vec{A}$ denotes the gauge field and $e$ is the electron charge. In a magnetic field the spectrum for a single Dirac cone is given by

$$
\begin{aligned}
E_{ \pm}(m) & = \pm \omega_{c} \sqrt{m} \\
\text { with } \omega_{c} & =v_{F} \sqrt{2 e B},
\end{aligned}
$$

where $m$ is the Landau-level index. These Landau levels are highly degenerate and interestingly the zeroth Landau level only lives in one component of the spinor.

\section{B. Main questions and summary of the results}

The purpose of this paper is to investigate the applicability of the standard LK formula upon approaching the Dirac point. There are three aspects to this question which we investigate separately in Sec. II: 
(I) What is the effect of temperature as the temperature approaches the value of the chemical potential in graphene?

(II) How is this modified in the presence of disorder?

(III) Is the effect of inelastic processes also to just modify the effective mass or do we have an additional damping term due to interaction effects associated with another inelastic Dingle damping temperature? The main results of the paper regarding these questions are as follows:

(I) Temperature acts in a similar manner as in standard twodimensional electron gases. However, there is an interesting modification upon approaching the Dirac point, which is, that temperature modifies the oscillation frequency, meaning the oscillation frequency is not a pure geometrical quantity anymore. Temperature also provides a cutoff for the quantum oscillations meaning that as soon as temperature is on the order of the chemical potential the quantum oscillations die altogether.

(II) The effect of disorder can be described in the same way as in the two-dimensional electron gas, accounted for by a Dingle temperature. In Dirac systems, however, there also is a damping term due to the coupling of temperature and disorder. Furthermore, disorder in graphene also affects the oscillation frequency, which is not the case in a $2 \mathrm{DEG}$.

(III) Unlike in a $2 \mathrm{DEG}$, electron-electron interactions in graphene lead to renormalization effects and inelastic effects.

We performed all our calculations for the specific case of graphene, but the results are also applicable to other Dirac systems without any restrictions. The only specification is a factor of 4 which is a consequence of the spin and valley degeneracy in graphene.

\section{THE OSCILLATORY GRAND POTENTIAL}

Our starting point is the Luttinger-Ward functional [9] which relates the thermodynamic potential $\Omega$ of the system to its Green's function, $\hat{G}$,

$$
\Omega=-T \operatorname{Tr} \ln \left(-\hat{G}^{-1}\right)-T \operatorname{Tr}(\hat{G} \hat{\Sigma})+\Omega^{\prime} .
$$

This expression is the starting point to determine all oscillating thermodynamic quantities such as, for instance, the magnetization or the specific heat. The Green's function for graphene in a magnetic field reads

$$
\hat{G}_{m}^{-1}\left(i \omega_{n}\right)=\left(\begin{array}{cc}
i \omega_{n}+\mu & \omega_{c} \sqrt{m} \\
\omega_{c} \sqrt{m} & i \omega_{n}+\mu
\end{array}\right)-\hat{\Sigma}\left(i \omega_{n}, m\right) .
$$

(We set the Boltzmann constant, $k_{B} \equiv 1$, as well as Planck's constant, $\hbar \equiv 1$.) The trace implies summation over the Landau-level index $m$, the fermonic Matsubara frequencies $\omega_{n}=\pi T(2 n+1)$, and the different degenerate states within one Landau level. The self-energy $\hat{\Sigma}$ accounts for disorder $\left(\hat{\Sigma}_{\text {dis }}\right)$ or electron-electron interactions $\left(\hat{\Sigma}_{e e}\right)$. The terms $T \operatorname{Tr}(\hat{G} \hat{\Sigma})$ and $\Omega^{\prime}$ are introduced to avoid overcounting of diagrams. Their oscillatory parts cancel each other [13] such that the magnetic oscillations are fully described by

$$
\Omega_{\mathrm{mo}}=-T \operatorname{Tr} \ln \left(-\hat{G}^{-1}\right)=-D T \sum_{m} \sum_{\omega_{n}} \ln \left[-g_{m}^{-1}\left(i \omega_{n}\right)\right],
$$

where $g_{m}^{-1}$ are the eigenvalues of the matrix $\hat{G}^{-1}$. The factor $D$ accounts for the sum over degenerate Landau levels,

$$
D=\frac{e B L^{2}}{\hbar \pi}=\frac{\omega_{c}^{2} L^{2}}{2 \pi v_{F}^{2}},
$$

where $L$ is the size of the system. We use the Poisson summation formula which relates the summation of a function to the function's continuous integral:

$$
\begin{aligned}
\sum_{m=0}^{\infty} f_{m} & =\lim _{\epsilon \rightarrow 0^{+}} \sum_{m=-\infty}^{\infty} \int_{-\epsilon}^{\infty} d x f(x) \delta(x-m) \\
& =\sum_{l=-\infty}^{\infty} \int_{0}^{\infty} d x f(x) e^{i 2 \pi l x} \\
& =\int_{0}^{\infty} d x f(x)+2 \sum_{l=1}^{\infty} \int_{0}^{\infty} d x f(x) \cos 2 \pi l x .
\end{aligned}
$$

This approach is advantageous when the Landau levels are sufficiently broadened due to, e.g., disorder or temperature which we assume throughout this paper. In this case we obtain a small parameter, $\omega_{c} / \gamma(\gamma$ is the disorder potential $)$ and $\omega_{c} / T$, respectively, such that the series can be truncated.

The first term describes the $B=0$ state and consequently does not contribute to the oscillations. We insert the function defined in Eq. (5) into Eq. (7) and integrate by parts,

$$
\begin{aligned}
\Omega_{\mathrm{mo}^{\prime}}= & -2 D T \sum_{\omega_{n}} \sum_{l=1}^{\infty} \int_{-\epsilon}^{\infty} d x \ln \left[-g^{-1}\left(x, i \omega_{n}\right)\right] \cos 2 \pi l x \\
= & -2 D T \sum_{\omega_{n}} \sum_{l=1}^{\infty}\left[\ln \left[-g^{-1}\left(x, i \omega_{n}\right)\right] \frac{\sin (2 \pi l x)}{2 \pi l}\right]_{0}^{\infty} \\
& +2 D T \sum_{\omega_{n}} \sum_{l=0}^{\infty} \int_{0}^{\infty} \frac{1}{-g^{-1}\left(x, i \omega_{n}\right)} \frac{d}{d x}\left[-g^{-1}\left(x, i \omega_{n}\right)\right] \\
& \times \frac{\sin (2 \pi l x)}{2 \pi l} d x .
\end{aligned}
$$

The first term is nonoscillatory and finite due to a cutoff in the Green's function. The oscillatory part consequently reads

$$
\begin{aligned}
\tilde{\Omega}= & 2 D T \sum_{\omega_{n}} \sum_{l=0}^{\infty} \int_{0}^{\infty} \frac{1}{-g^{-1}\left(x, i \omega_{n}\right)} \frac{d}{d x}\left[-g^{-1}\left(x, i \omega_{n}\right)\right] \\
& \times \frac{\sin (2 \pi l x)}{2 \pi l} d x .
\end{aligned}
$$

With the following ansatz for the Green's function

$$
\hat{G}^{-1}=\left(\begin{array}{ll}
g_{1} & g_{2} \\
g_{2} & g_{1}
\end{array}\right)
$$

which has eigenvalues

$$
g^{-1}=g_{1} \pm\left|g_{2}\right|,
$$

we find

$$
\tilde{\Omega}=\frac{D T}{\pi} \sum_{\omega_{n}} \sum_{l=1}^{\infty} \frac{1}{l} \int_{0}^{\infty}\left[\frac{\sin (2 \pi l x) \frac{d}{d x}\left(g_{1}^{2}-g_{2}^{2}\right)}{g_{1}^{2}-g_{2}^{2}}\right] d x .
$$


In the following Secs. II A-IIC, we specify this generic expression according to the Green's function of the discussed system.

\section{A. The clean limit}

In the limit of a clean system the Green's function is simply the free Green's function, meaning we have

$$
\hat{G}_{m}^{0-1}\left(i \omega_{n}\right)=\left(\begin{array}{cc}
i \omega_{n}+\mu & \omega_{c} \sqrt{m} \\
\omega_{c} \sqrt{m} & i \omega_{n}+\mu
\end{array}\right) .
$$

Inserting this Green's function into the oscillatory potential, Eq. (12), we find

$\tilde{\Omega}=-\frac{\omega_{c}^{2} D T}{\pi} \sum_{\omega_{n}} \sum_{l=1}^{\infty} \frac{1}{l} \int_{0}^{\infty}\left[\frac{\sin (2 \pi l x)}{\left(i \omega_{n}+\mu\right)^{2}-\omega_{c}^{2} x}\right] d x$.

The integral can be computed using residue theorem and the calculation is performed in Appendix A. We obtain

$$
\begin{aligned}
\tilde{\Omega}_{\mathrm{osc}}= & \frac{2 T \omega_{c}^{2} L^{2}}{\pi v_{F}^{2}} \sum_{l=1}^{\infty} \sum_{\omega_{n}>0}^{|\mu|} \frac{e^{-\left(4 \pi l / \omega_{c}^{2}\right) \omega_{n}|\mu|}}{l} \\
& \times \cos \left(\frac{2 \pi l\left(\mu^{2}-\omega_{n}^{2}\right)}{\omega_{c}^{2}}\right) .
\end{aligned}
$$

We will come back to a discussion of this expression in Sec. III.

\section{B. Weak disorder limit}

We do not attempt to make a realistic modeling of the properties of graphene with disorder but instead stick to the most simple treatment of disorder within the self-consistent Born approximation along the lines of Ref. [17]. The disorder induced self-energy is explicitly computed in Appendix B. In the limit of weak magnetic field (i.e., not well separated Landau levels) and white noise disorder, $\omega_{c} \ll \gamma$ ( $\gamma$ is the strength of the disorder potential), the self-energy can be assumed as

$$
\begin{aligned}
\hat{\Sigma}_{\mathrm{dis}} & =-\gamma\left(i \omega_{n}-\mu\right) \ln \left(\frac{v_{F}^{2} \Lambda^{2}-\left(i \omega_{n}-\mu\right)^{2}}{-\left(i \omega_{n}-\mu\right)^{2}}\right) \mathbb{1} \\
& \approx-\gamma\left(i \omega_{n}-\mu\right) \ln \left(\frac{v_{F}^{2} \Lambda^{2}}{-\left(i \omega_{n}-\mu\right)^{2}}\right) \mathbb{1},
\end{aligned}
$$

where $v_{F}$ is the Fermi velocity and $\Lambda$ is a high-energy cutoff. This self-energy is diagonal and independent of energy. Consequently the oscillatory potential, Eq. (12), simplifies to

$$
\tilde{\Omega}=-\frac{\omega_{c}^{2} D T}{\pi} \sum_{\omega_{n}} \sum_{l=1}^{\infty} \frac{1}{l} \int_{0}^{\infty} d x \frac{\sin (2 \pi l x)}{\left(i \omega_{n}+\mu-\Sigma_{\mathrm{dis}}\right)^{2}-\omega_{c}^{2} x} .
$$

We obtain

$$
\begin{aligned}
\tilde{\Omega}_{\mathrm{osc}}= & -\frac{2 T \omega_{c}^{2} L^{2}}{\pi v_{F}^{2}} \sum_{l=1}^{\infty} \frac{1}{l} \\
& \times \sum_{\omega_{n}>0}^{|\mu| /[1+\gamma(\pi / 2)]} e^{-\left(4 \pi l / \omega_{c}^{2}\right)\left[\left(\mu^{2}-\omega_{n}^{2}\right)(\pi \gamma-2 \phi \gamma)+|\mu| \omega_{n}(1+2 \gamma \Gamma)\right]}
\end{aligned}
$$

$$
\begin{aligned}
& \times \cos \left(\frac { 2 \pi l } { \omega _ { c } ^ { 2 } } \left[\left(\mu^{2}-\omega_{n}^{2}\right)(1+2 \gamma \Gamma)\right.\right. \\
&\left.\left.+4 \gamma|\mu| \omega_{n}(2 \phi-\pi)\right]\right) \\
& \text { with } \quad \Gamma=\ln \left(\frac{\left(v_{F} \Lambda\right)^{2}}{\omega_{n}^{2}+\mu^{2}}\right) \text { and } \phi=\arctan \left(\frac{\omega_{n}}{|\mu|}\right) .
\end{aligned}
$$

The concrete computation of the integral is performed in Appendix $\mathrm{C}$ and a discussion is again given in Sec. III.

\section{The effect of inelastic scattering on magnetooscillations}

Within this section we investigate the effect of interactions in perturbation theory. We first calculate the oscillatory part of the grand potential with an ansatz for an energy dependent self-energy $\hat{\Sigma}_{e e}$. Then we will compute the interaction induced self-energy for graphene to second order in perturbation theory. We will perform this calculation in $k$ space and for zero chemical potential. This is a strong simplification and we will comment on it later. We will see that for finite temperatures the imaginary part contributes an additional damping factor to the LK amplitude.

We make the following ansatz for the self-energy, which we will motivate below by means of an explicit calculation, whose details can be found in Appendix D:

$$
\begin{aligned}
\hat{\Sigma}_{e e}\left(m, i \omega_{n}\right)= & \left(\begin{array}{cc}
i \omega_{n}\left(1-Z^{-1}\right) & \left(1-Z_{v_{F}}\right) \omega_{c} \sqrt{m} \\
\left(1-Z_{v_{F}}\right) \omega_{c} \sqrt{m} & i \omega_{n}\left(1-Z^{-1}\right)
\end{array}\right) \\
& +\left(\begin{array}{cc}
i \delta \Sigma^{\prime \prime}\left(\omega_{c} \sqrt{m}, \omega_{n}\right) & \delta \Sigma^{\prime}\left(\omega_{c} \sqrt{m}, \omega_{n}\right) \\
\delta \Sigma^{\prime}\left(\omega_{c} \sqrt{m}, \omega_{n}\right) & i \delta \Sigma^{\prime \prime}\left(\omega_{c} \sqrt{m}, \omega_{n}\right)
\end{array}\right) .
\end{aligned}
$$

Here, we assumed that $Z$ and $Z_{v_{F}}$ account for logarithmic renormalizations and do not explicitly depend on energy. $\delta \Sigma^{\prime \prime}$ and $\delta \Sigma^{\prime}$ are real and correspond to nonlogarithmic contributions which potentially depend on temperature. The Green's function reads

$$
\begin{aligned}
\hat{G}^{-1}\left(m, i \omega_{n}\right)= & \left(\begin{array}{cc}
\left(i \omega_{n}+\mu\right) Z^{-1} & Z_{v_{F}} \omega_{c} \sqrt{m} \\
Z_{v_{F}} \omega_{c} \sqrt{m} & \left(i \omega_{n}+\mu\right) Z^{-1}
\end{array}\right) \\
& -\left(\begin{array}{cc}
i \delta \Sigma^{\prime \prime}\left(\omega_{c} \sqrt{m}, \omega_{n}\right) & \delta \Sigma^{\prime}\left(\omega_{c} \sqrt{m}, \omega_{n}\right) \\
\delta \Sigma^{\prime}\left(\omega_{c} \sqrt{m}, \omega_{n}\right) & i \delta \Sigma^{\prime \prime}\left(\omega_{c} \sqrt{m}, \omega_{n}\right)
\end{array}\right) .
\end{aligned}
$$

The eigenvalues of this matrix are

$$
g_{m}^{-1}=\left(i \omega_{n}+\mu\right) Z^{-1}-i \delta \Sigma^{\prime \prime} \mp\left(\sqrt{m} \omega_{c} Z_{v_{F}}-\delta \Sigma^{\prime}\right) .
$$

We linearize the denominator of Eq. (12) around the pole $x_{0}$ with $g_{1}^{2}-\left.g_{2}^{2}\right|_{x=x_{0}}=0$,

$$
\tilde{\Omega}=\frac{D T}{\pi} \sum_{\omega_{n}} \sum_{l=1}^{\infty} \frac{1}{l} \int_{0}^{\infty}\left[\frac{\sin (2 \pi l x) \frac{d}{d x}\left(g_{1}^{2}-g_{2}^{2}\right)}{\left.\left(x-x_{0}\right) \frac{d}{d x}\left(g_{1}^{2}-g_{2}^{2}\right)\right|_{x=x_{0}}}\right] d x .
$$

We expand the pole in powers of the interaction parameter $\alpha$, which we will define below ( $\delta \Sigma^{\prime}$ and $\delta \Sigma^{\prime \prime}$ are quadratic in $\alpha$ ), 
and write

$$
x_{0} \approx x_{0}^{(0)}+x_{0}^{(2)}
$$

To lowest order we have

$$
\omega_{c}^{2} Z_{v_{F}}^{2} x_{0}^{(0)}=\left(i \omega_{n}+\mu\right)^{2} Z^{-2} .
$$

$$
\begin{aligned}
\tilde{\Omega}_{\mathrm{osc}}= & 2 D T \sum_{l=1}^{\infty} \frac{1}{l} \sum_{\omega_{n}>0}^{\approx|\mu|} e^{-\left(4 \pi l / \omega_{c}^{2} Z_{v_{F}}^{2}\right)\left[\omega_{n} \mu Z^{-2}+\left(\operatorname{Im}\left\{\delta \Sigma^{\prime \prime}\right\}+\operatorname{Re}\left\{\delta \Sigma^{\prime}\right\}\right) \omega_{n} Z^{-1}+\left(\operatorname{Im}\left\{\delta \Sigma^{\prime}\right\}-\operatorname{Re}\left\{\delta \Sigma^{\prime \prime}\right\}\right) \mu Z^{-1}\right]} \\
& \times \cos \left(\frac{2 \pi l}{\omega_{c}^{2} Z_{v_{F}}^{2}}\left[\left(-\omega_{n}^{2}+\mu^{2}\right) Z^{-2}+2\left(\operatorname{Re}\left\{\delta \Sigma^{\prime \prime}\right\}-\operatorname{Im}\left\{\delta \Sigma^{\prime}\right\}\right) \omega_{n} Z^{-1}+2\left(\operatorname{Im}\left\{\delta \Sigma^{\prime \prime}\right\}+\operatorname{Re}\left\{\delta \Sigma^{\prime}\right\}\right) \mu Z^{-1}\right]\right)
\end{aligned}
$$

In Appendix D we compute the interaction induced selfenergy to second order in the fine-structure constant $\alpha=$ $e^{2} /\left(4 \pi \epsilon_{0} \epsilon_{r} v_{F}\right)\left(\epsilon_{0}\right.$ being the vaccuum dielectric constant and $\epsilon_{r}$ the relative permittivity). We perform the calculation in $k$ space and not in the Landau-level basis. This approach is questionable, for instance, in light of the well-known magnetic catalysis in systems with Dirac fermions in a magnetic field [18]. However, we believe that this approach is justified in the limit of sufficient thermal Landau-level broadening, $T>\omega_{c}$, since in that limit the "effective" density of states which enters the computation is closer to the one without a magnetic field than to the singular one with the Landau levels. To lowest order in $\alpha$ we find

$$
\hat{\Sigma}_{e e}^{(1)}\left(\mathbf{k}, i \omega_{n}\right)=\frac{\alpha v_{F} \mathbf{k} \hat{\boldsymbol{\sigma}}}{4} \ln \left(\frac{4 \Lambda}{v_{F} k}\right) .
$$

This term has no information about real inelastic scattering processes which is why we go to two-loop order. A systematic expansion is beyond the scope of this paper, which is why we concentrate on a class of diagrams. Concretely, to second order we only consider the random phase approximation (RPA) type diagram which scales linearly with the number of flavors $N$ and discard the crossed diagrams. Philosophically, one could say that we expand perturbatively in $\alpha$ assuming that $N \gg 1$ while $\alpha N \ll 1$ (note, however, that we do not perform a systematic $1 / N$ expansion since we are not resumming the whole RPA series).

$$
\begin{aligned}
\hat{\Sigma}_{e e}^{(2)}\left(\mathbf{k}, i \omega_{n}\right)= & -\frac{\alpha^{2}}{2}\left(i \omega_{n} \mathbb{1}+v_{F} \mathbf{k} \hat{\boldsymbol{\sigma}}\right) \\
& \times\left[\frac{1}{6} \ln \left(\frac{\Lambda^{2} v_{F}^{2}}{k^{2} v_{F}^{2}+\omega_{n}^{2}}\right)-\frac{1}{3} \ln (2)+\frac{5}{9}\right] .
\end{aligned}
$$

Within renormalized perturbation theory we can define the renormalization $Z$ factors we introduced in the ansatz for the self-energy, Eq. (20) (note that we use a nonstandard definition). We only include the logarithmically dependent parts since the other parts are irrelevant for the flow equations. They read

$$
\begin{aligned}
& Z^{-1}=1+\frac{\alpha^{2}}{12} \ln \frac{\Lambda^{2} v_{F}^{2}}{v_{F}^{2} k^{2}+\omega_{n}^{2}}, \\
& Z_{v_{F}}=1+\frac{\alpha}{4} \ln \frac{4 \Lambda}{v_{F} k}-\frac{\alpha^{2}}{12} \ln \frac{\Lambda^{2} v_{F}^{2}}{v_{F}^{2} k^{2}+\omega_{n}^{2}}+\frac{\alpha^{2}}{16} \ln ^{2} \frac{4 \Lambda}{v_{F} k} .
\end{aligned}
$$

To quadratic order we find

$$
\begin{aligned}
\omega_{c}^{2} Z_{v_{F}}^{2} x_{0}^{(2)}= & 2 i \delta \Sigma^{\prime \prime}\left(-i \omega_{n}-\mu\right) Z^{-1} \\
& +2 \delta \Sigma^{\prime}\left(x_{0}^{(0)}\right) \sqrt{x_{0}^{(0)}} \omega_{c} Z_{v_{F}} .
\end{aligned}
$$

Computing the integral using residue theorem, we obtain

While $Z$ corresponds to the field renormalization renormalizing $\omega_{n}, Z_{v_{F}}$ renormalizes $\omega_{c}$ and can thus be identified with the renormalization factor of the Fermi velocity [cf. Eq. (3)]. The last term in $Z_{v_{F}}$ with the square logarithm results from doing renormalized perturbation theory on the two-loop level: it can be traced back to including a counterterm containing the one-loop result into the perturbation theory. Overall, the Green's function reads

$$
\hat{G}^{-1}\left(\mathbf{k}, i \omega_{n}\right)=Z^{-1}\left[(i \omega+\mu) \mathbb{1}-v_{F} \mathbf{k} \hat{\boldsymbol{\sigma}} Z Z_{v_{F}}\right]
$$

and consequently we can define a renormalized Fermi velocity $v_{F}^{R}$ as

$$
v_{F}^{R}=v_{F} Z Z_{v_{F}}
$$

where $v_{F}$ is the bare Fermi velocity. Exploiting $\frac{d v_{F}}{d \ln \Lambda}=0$ we obtain the flow of the renormalized Fermi velocity as

$$
\begin{aligned}
\frac{d v_{F}^{R}}{d \ln \Lambda} & =v_{F}\left(\frac{\partial Z Z_{v_{F}}}{\partial \ln \Lambda}+\frac{\partial Z Z_{v_{F}}}{\partial \alpha} \frac{\partial \alpha}{\partial v_{F}^{R}} \frac{\partial v_{F}^{R}}{\partial \ln \Lambda}\right) \\
& =\frac{v_{F}^{R}}{Z Z_{v_{F}}}\left(\frac{\partial Z Z_{v_{F}}}{\partial \ln \Lambda}-\frac{\alpha}{Z Z_{v_{F}}} \frac{\partial Z Z_{v_{F}}}{\partial \alpha} \frac{\partial Z_{v_{F}}}{\partial \ln \Lambda}\right) \\
& =v_{F}^{R}\left(\frac{\alpha}{4}-\frac{\alpha^{2}}{3}\right)+O\left(\alpha^{3}\right) .
\end{aligned}
$$

The flow of $\alpha$ itself to lowest order in perturbation theory (one loop) is given by $\frac{d \alpha}{d \ln \Lambda}=-\frac{\alpha^{2}}{4}$. This means renormalizations of $\alpha$ itself do not interfere with this result since they are of higher order in $\alpha$ leading to lowest-order contributions at $O\left(\alpha^{3}\right)$. The flow equation implies a critical $\alpha_{c}=3 / 4$, which potentially describes a repulsive critical point separating weak coupling from strong coupling. Under our assumption that $N \alpha \ll 1$ this critical point is outside the validity of a perturbative expansion and therefore is not to be trusted. In fact, in a strict large- $N$ limit to all orders in $\alpha$ the absence of such a critical point was shown by Son in Refs. [4,19].

For additional effects of inelastic scattering we have also investigated $\hat{\Sigma}^{(2)}$ at finite temperatures. Here, we concentrate our discussion on the diagonal part of $\hat{\Sigma}^{(2)}$, called $\delta \Sigma^{\prime \prime}=$ $\Sigma_{\text {diag }}^{(2)}(T)-\Sigma_{\text {diag }}^{(2)}(T=0)$, which enters the amplitude and frequency of the oscillation [see Eq. (26)] and was introduced 
in the ansatz for the self-energy (20). We have found that

$$
\begin{aligned}
\delta \Sigma^{\prime \prime}\left(\mathbf{k}, \omega_{n}\right)= & -\frac{\alpha^{2} \pi \ln 2}{12} \omega_{n} \\
& \times\left(\frac{T^{2}}{\omega_{n}^{2}+v_{F}^{2} k^{2}}+O\left\{\left(\frac{T^{2}}{\omega_{n}^{2}+v_{F}^{2} k^{2}}\right)^{2}\right\}\right),
\end{aligned}
$$

in an expansion of the integral (D12) in $T^{2} /\left(\omega_{n}^{2}+v_{F}^{2} k^{2}\right)$.

\section{DISCUSSION}

In this section we give an overview of our main results and compare them to the more standard 2DEG. The derivation of the LK formula for the 2DEG including interactions was performed in Ref. [13] which is what we base our results on. In order to be able to compare the expressions we express all quantities in terms of electronic densities and the magnetic field (instead of chemical potential and cyclotron frequency). In the case of Dirac theory the electronic density at zero temperature is related to the chemical potential $\mu$ via $n_{\text {Dirac }}=\frac{\mu^{2}}{4 \pi v_{F}^{2}}$, while for the $2 \mathrm{DEG}$ it is related via $n_{2 \mathrm{DEG}}=\frac{m \mu}{2 \pi}$ (note that these expressions still have to be multiplied with the appropriate degeneracy factors for spin and valley/flavor). Equivalently, the cyclotron frequency in the case of a Dirac theory reads $\omega_{c}$ Dirac $=v_{F} \sqrt{2 e B}$, while for the $2 \mathrm{DEG}$ it is given by $\omega_{c} 2 \mathrm{DEG}=\frac{e B}{m}$, where $m$ denotes the electronic band mass of the 2DEG.

\section{A. The Lifshitz-Kosevich formula without interactions}

In Table I we discuss the Lifshitz-Kosevich formula for the 2DEG [13] and for a Dirac theory with and without disorder in the absence of interaction effects. We observe two peculiarities: (a) In contrast to the oscillations in the 2DEG, the oscillations in a Dirac theory die above a characteristic temperature $T_{\text {osc }}$. This temperature depends on the strength of the scattering potential parametrized by $\gamma$ and is given by $\pi T_{\mathrm{osc}}=\frac{|\mu|}{1+\gamma \frac{\pi}{2}}$. (b) The effective oscillation frequency in a Dirac theory is not only a geometric quantity but instead also depends on temperature itself via the dependence upon $\omega_{n}$. Disorder in a Dirac theory, unlike in the 2DEG, also affects the oscillation frequency.

In the Fermi-liquid regime of a Dirac theory, i.e., at $\mu \gg T$, a slightly modified formula for a "standard" 2 DEG is obtained where the only modification sits in the temperature damping factor.

The difference between a Dirac theory and the 2DEG becomes pronounced upon approaching the Dirac point, meaning if the temperature becomes comparable to the chemical potential. This is shown in Fig. 1 where a comparison in the clean noninteracting system at finite temperature is shown.

In Table I we also give an expression for the Dingle temperature for the 2DEG as well as for a Dirac material. We see, that in graphene, the Dingle temperature also depends on temperature, meaning there is an additional damping term due to the coupling of temperature and impurity scattering, which does not occur in the 2DEG. In Table I we also give
TABLE I. Comparison of LK formula in 2DEG and a Dirac theory in the clean and disordered case.

$$
\begin{aligned}
& \text { 2DEG } \\
& \tilde{\Omega}_{\mathrm{osc}}=\frac{e B L^{2}}{\pi} T \sum_{l=1}^{\infty} \sum_{\omega_{n}>0} \frac{(-1)^{l}}{l} e^{-(2 \pi l m / e B)\left[\omega_{n}+(1 / 2 \tau)\right]} \cos \left(\frac{4 \pi^{2} l n}{e B}\right), \\
& T_{D}=\frac{1}{2 \pi \tau}, \\
& \lim _{T_{D} \rightarrow 0} \tilde{\Omega}_{\mathrm{osc}}=\frac{e B L^{2}}{\pi} T \sum_{l=1}^{\infty} \frac{(-1)^{l}}{l} \frac{1}{\sinh \left(\frac{2 \pi^{2} l m T}{e B}\right)} \cos \left(\frac{4 \pi^{2} l n}{e B}\right) . \\
& \tilde{\Omega}_{\mathrm{osc}}=-\frac{e B L^{2}}{\pi} T \sum_{l=1}^{\infty} \frac{1}{l} \sum_{\omega_{n}>0}^{|\mu| /[1+\gamma(\pi / 2)]} \\
& \times e^{-4 \pi l\left\{\left[(2 \pi / n e B)-\left(\omega_{n}^{2} / 2 v_{F}^{2} e B\right)\right](\pi \gamma-2 \phi \gamma)+\sqrt{\pi n \omega_{n}^{2} /\left(v_{F} e B\right)^{2}}(1+2 \gamma \Gamma)\right\}} \\
& \times \cos \left\{2 \pi l\left[\left(\frac{2 \pi n}{e B}-\frac{\omega_{n}^{2}}{2 v_{F}^{2} e B}\right)(1+2 \gamma \Gamma)-\Phi\right]\right\}, \\
& \Phi=4 \gamma \sqrt{\frac{\pi n \omega_{n}^{2}}{\left(v_{F} e B\right)^{2}}}(\pi-2 \phi), \\
& T_{D}=\omega_{n}\left(\frac{\left(\mu^{2}-\omega_{n}^{2}\right)}{|\mu| \omega_{n}}(\pi \gamma-2 \phi \gamma)+2 \gamma \Gamma\right) \text {, } \\
& T_{D}(T=0)=|\mu| \pi \gamma \text {, } \\
& \lim _{T_{D} \rightarrow 0} \tilde{\Omega}_{\mathrm{osc}}=\frac{e B L^{2}}{\pi} T \sum_{l=1}^{\infty} \sum_{\omega_{n}>0}^{|\mu|} \frac{1}{l} e^{-4 \pi l} \sqrt{\pi n \omega_{n}^{2} /\left(v_{F} e B\right)^{2}} \\
& \times \cos \left[2 \pi l\left(\frac{2 \pi n}{e B}-\frac{\omega_{n}^{2}}{2 v_{F}^{2} e B}\right)\right], \\
& \mu \gg T \text {, } \\
& \tilde{\Omega}_{\mathrm{osc}} \approx \frac{e B L^{2}}{2 \pi} \sum_{l=1}^{\infty} \frac{T}{l \sinh \left(4 \pi^{5 / 2} l T \frac{\sqrt{n}}{v_{F} e B}\right)} \cos \left(\frac{4 \pi^{2} l n}{e B}\right) .
\end{aligned}
$$

an account of the Dingle temperature for zero temperature, $T_{D}(T=0)$.

\section{B. The effect of electron-electron interactions on the Lifshitz-Kosevich formula}

In Table II we contrast the LK formula for weak interactions in a Dirac theory and in the 2DEG [13]. In contrast to Table I we go back to a notation in which we use chemical potential and the respective cyclotron frequencies. One of the main features of the 2DEG is that inelastic processes on the first Matsubara mode do not lead to an additional damping Dingle temperature

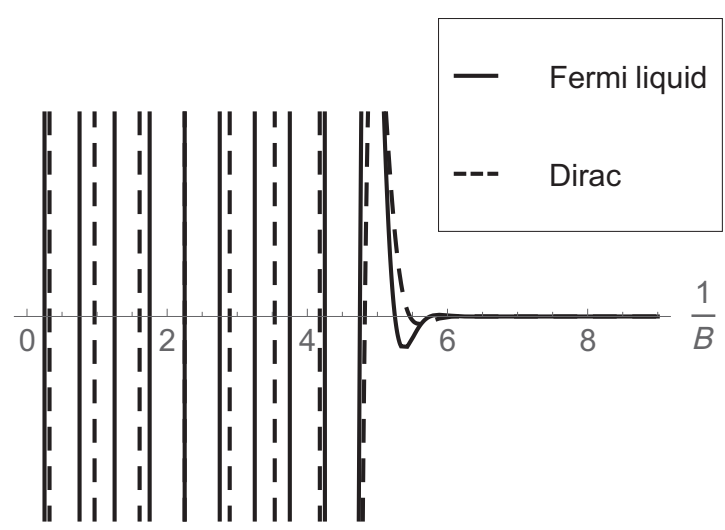

FIG. 1. Comparison of the standard LK formula adapted to a Dirac system (full line) to the modified LK formula in a clean and interaction-free system (dashed line). The parameter for $T / \mu=0.15$. We observe the absence of one clear frequency (amplitudes are not shown) for the modified formula which stems from the fact that we are in a regime where $T$ is not much smaller than $\mu$. 
TABLE II. The LK formula with electron-electron interactions.

\begin{tabular}{|c|c|}
\hline $2 \mathrm{DEG}$ & $\tilde{\Omega}_{\mathrm{osc}}=-\frac{e B L^{2}}{\pi} T \sum_{\omega_{n}>0} \sum_{l=1}^{\infty} \frac{1}{l} e^{-\left\{2 \pi l\left[\omega_{n}\left(1+\alpha_{0}\right)-\delta \Sigma^{\prime \prime}\right]\right\} / \omega_{c} 2 \mathrm{DEG}(1+\beta)} \cos \left(\frac{2 \pi l \tilde{\mu}}{\omega_{c} 2 \mathrm{DEG}(1+\beta)}\right)$ \\
\hline Dirac & $\begin{aligned} \tilde{\Omega}_{\mathrm{osc}}= & \frac{e B L^{2}}{\pi} T \sum_{l=1}^{\infty} \frac{1}{l} \sum_{\omega_{n}>0}^{\approx|\mu|} e^{-4 \pi l / \omega_{c}^{2} \operatorname{Dirac} Z_{v_{F}}^{2}\left(\omega_{n} \mu Z^{-2}+\left[\operatorname{Im}\left\{\delta \Sigma^{\prime \prime}\right\}+\operatorname{Re}\left\{\delta \Sigma^{\prime}\right\}\right] \omega_{n} Z^{-1}+\left[\operatorname{Im}\left\{\delta \Sigma^{\prime}\right\}-\operatorname{Re}\left\{\delta \Sigma^{\prime \prime}\right\}\right] \mu Z^{-1}\right)} \\
& \times \cos \left(\frac{2 \pi l}{\omega_{c \text { Dirac }}^{2} Z_{v_{F}}^{2}}\left[\left(-\omega_{n}^{2}+\mu^{2}\right) Z^{-2}+2\left(\operatorname{Re}\left\{\delta \Sigma^{\prime \prime}\right\}-\operatorname{Im}\left\{\delta \Sigma^{\prime}\right\}\right) \omega_{n} Z^{-1}+2\left(\operatorname{Im}\left\{\delta \Sigma^{\prime \prime}\right\}+\operatorname{Re}\left\{\delta \Sigma^{\prime}\right\}\right) \mu Z^{-1}\right]\right)\end{aligned}$ \\
\hline
\end{tabular}

[13]. This implies that interaction effects can fully be absorbed in renormalization factors.

The situation is different in graphene. Here, both inelastic effects as well as renormalization effects influence the amplitude. The inelastic effects are expressed by $\delta \Sigma^{\prime}$ and $\delta \Sigma^{\prime \prime}$. The latter is given by [cf. Eq. (33)]

$$
\begin{aligned}
\delta \Sigma^{\prime \prime}\left(x_{0}^{(0)}, i \omega_{n}\right) & \\
= & -\frac{\alpha^{2} \pi \ln 2}{12} \omega_{n}\left(\frac{T^{2}}{\left(\omega_{n}^{2}+\mu^{2}\right)\left(1-Z^{-2}\right)+2 i \omega_{n} Z^{-2} \mu}\right. \\
& \left.+O\left\{\left(\frac{T^{2}}{\left(\omega_{n}^{2}+\mu^{2}\right)\left(1-Z^{-2}\right)+2 i \omega_{n} Z^{-2} \mu}\right)^{2}\right\}\right) .
\end{aligned}
$$

However, unlike in the case of disorder, the inelastic effects vanish for zero temperature, as one should expect.

Another interesting property is that the dominant damping term

$$
e^{-4 \pi \omega_{n} \mu l / \omega_{c}^{2} Z_{v_{F}}^{2} Z^{2}}=e^{-4 \pi \omega_{n} \mu l /\left(\omega_{c}^{R}\right)^{2}}
$$

is fully accounted for by renormalizations of the Fermi velocity or cyclotron frequency. So this result is in agreement with a recent analysis, Ref. [5], carried out for Shubnikov-de Haas oscillations.

\section{CONCLUSION}

We derived a full quantitative expression which describes the de Haas-van Alphen oscillations in clean, disordered, and interacting Dirac systems. In the Fermi-liquid regime of the system we reproduce the standard Lifshitz-Kosevich formula for the two-dimensional electron gas, despite the differences due to a different dispersion, which yields $m=\frac{\mu}{v_{F}^{2}}$ for the cyclotron mass in Dirac systems. However, when approaching the Dirac point, we found two new features in the clean system: first, the frequency is not only a geometric quantity but instead it also depends on temperature itself; and second, the oscillations completely die as soon as $\mu<\pi T$. In the case of a disordered system, we find that disorder also affects the oscillation frequency and that there is an additional damping term due to the coupling of temperature and impurity scattering.

Most interesting is the effect of electron-electron interactions on the oscillation amplitude. We find that electron- electron interactions in Dirac systems damp the oscillations in two ways. They renormalize the Fermi velocity and to two-loop order they lead to an additional damping factor. This damping factor is absent in the more standard twodimensional electron gas. In an extension of this work, it would be very interesting to see whether our results for the LK formula would also survive for the case of Shubnikov-de Haas oscillations.

\section{ACKNOWLEDGMENTS}

We acknowledge discussions with A. Rosch and I. Herbut as well as financial support from the DFG FR 2627/3-1. This work is part of the D-ITP consortium, a program of the Netherlands Organization for Scientific Research (NWO) that is funded by the Dutch Ministry of Education, Culture and Science (OCW).

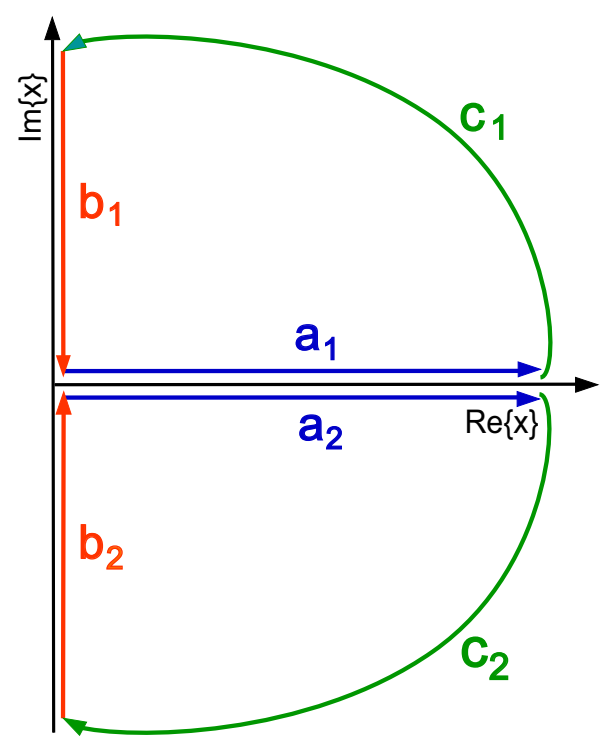

FIG. 2. Integration path: we use the integration path 1 in the upper complex half-plane to evaluate the integral $\frac{e^{i 2 \pi l x}}{\left(i \omega_{n}+\mu\right)^{2}-\omega_{c}^{2} x}$ and path 2 in the lower complex half-plane for the integral $\frac{e^{-i 2 \pi l x}}{\left(i \omega_{n}+\mu\right)^{2}-\omega_{c}^{2} x}$ such that the paths $c_{1,2}$ vanish for $x \rightarrow \infty$. Thus the original integral along the real axis, paths $a_{1,2}$, can be written as an integral along the imaginary axis, paths $b_{1,2}$, plus summation over residues. 


\section{APPENDIX A: THE OSCILLATORY INTEGRAL FOR CLEAN GRAPHENE}

The integral of Eq. (14) can be evaluated using the residue theorem. The used integration path is described in Fig. 2.

$$
\begin{aligned}
\int_{0}^{\infty} d x \frac{\sin (2 \pi l x)}{\left(i \omega_{n}+\mu\right)^{2}-\omega_{c}^{2} x}= & \frac{1}{2 i} \int_{0}^{\infty} d x \frac{e^{i 2 \pi l x}-e^{-i 2 \pi l x}}{\left(i \omega_{n}+\mu\right)^{2}-\omega_{c}^{2} x} \\
= & \frac{1}{2} \int_{0}^{\infty} d x e^{-2 \pi l x}\left(\frac{1}{\left(i \omega_{n}+\mu\right)^{2}-i \omega_{c}^{2} x}+\frac{1}{\left(i \omega_{n}+\mu\right)^{2}+i \omega_{c}^{2} x}\right) \\
& -\frac{\pi}{\omega_{c}^{2}} \Theta\left(\mu^{2}-\omega_{n}^{2}\right)\left[e^{-4 \pi l \omega_{n} \mu / \omega_{c}^{2}} e^{i 2 \pi l\left(\mu^{2}-\omega_{n}^{2}\right) / \omega_{c}^{2}} \Theta\left(\omega_{n} \mu\right)+e^{4 \pi l \omega_{n} \mu / \omega_{c}^{2}} e^{-i 2 \pi l\left(\mu^{2}-\omega_{n}^{2}\right) / \omega_{c}^{2}} \Theta\left(-\omega_{n} \mu\right)\right] .
\end{aligned}
$$

If we use $\sum_{\omega_{n}} F\left(\omega_{n}\right)=\sum_{\omega_{n}>0}\left[F\left(\omega_{n}\right)+F\left(-\omega_{n}\right)\right]$ we get the following expression:

$$
\begin{aligned}
\sum_{\omega_{n}} & \int_{0}^{\infty} d x \frac{\sin (2 \pi l x)}{\left(i \omega_{n}+\mu\right)^{2}-\omega_{c}^{2} x} \\
= & \sum_{\omega_{n}>0}\left(\mu^{2}-\omega_{n}^{2}\right) \int_{0}^{\infty} d x e^{-2 \pi l x}\left(\frac{1}{\left(\mu^{2}-\omega_{n}^{2}\right)^{2}+\left(2 \omega_{n} \mu-\omega_{c}^{2} x\right)}+\frac{1}{\left(\mu^{2}-\omega_{n}^{2}\right)^{2}+\left(2 \omega_{n} \mu+\omega_{c}^{2} x\right)}\right) \\
& -\frac{\pi}{\omega_{c}^{2}} \sum_{\omega_{n}>0} e^{-4 \pi l \omega_{n}|\mu| / \omega_{c}^{2}} 2 \cos \left(\frac{2 \pi l}{\omega_{c}^{2}}\left(\mu^{2}-\omega_{c}^{2}\right)\right) \Theta\left(\mu^{2}-\omega_{n}^{2}\right)
\end{aligned}
$$

Importantly, the first term on the right-hand-side in Eq. (A2) does not contribute an oscillatory term to the thermodynamic potential.

\section{APPENDIX B: THE DISORDER INDUCED SELF-ENERGY}

It is well known that within the self-consistent Born approximation (SCBA) there is a difference between the self-energy on the different sublattices [17]. The defining equation reads

$$
\Sigma_{\mathrm{dis}}^{a(b)}=\alpha_{\mathrm{dis}} \omega_{c}^{2} \sum_{m} \frac{i \omega_{n}+\mu-\Sigma_{\mathrm{dis}}^{b(a)}}{\left(i \omega_{n}+\mu-\Sigma_{\mathrm{dis}}^{a}\right)\left(i \omega_{n}+\mu-\Sigma_{\mathrm{dis}}^{b}\right)-\omega_{c}^{2} m},
$$

where $\alpha_{\text {dis }}$ is a dimensionless parameter characterizing the strength of the disorder potential.

In the following we assume that sufficiently high-lying Landau levels are populated such that the asymmetry between the sublattices is irrelevant. We then have

$$
\Sigma_{\mathrm{dis}}=\alpha_{\mathrm{dis}} \omega_{c}^{2} \sum_{m=0}^{\infty} \frac{i \omega_{n}+\mu-\Sigma_{\mathrm{dis}}}{\left(i \omega_{n}+\mu-\Sigma_{\mathrm{dis}}\right)^{2}-\omega_{c}^{2} m}
$$

We use the Poisson summation formula, Eq. (7), to dualize the sum over Landau levels making the expression more amenable to approximations for weak fields.

$$
\begin{aligned}
\Sigma_{\mathrm{dis}}= & \alpha_{\mathrm{dis}} \omega_{c}^{2} \sum_{l=-\infty}^{\infty} \int_{0}^{v_{F} \Lambda / \omega_{c}} d x \frac{i \omega_{n}+\mu-\Sigma_{\mathrm{dis}}}{\left(i \omega_{n}+\mu-\Sigma_{\mathrm{dis}}\right)^{2}-\omega_{c}^{2} x} e^{i 2 \pi l x} \\
= & \alpha_{\mathrm{dis}} \int_{0}^{v_{F}^{2} \Lambda^{2}} d x \frac{i \omega_{n}+\mu-\Sigma_{\mathrm{dis}}}{\left(i \omega_{n}+\mu-\Sigma_{\mathrm{dis}}\right)^{2}-x} \\
& +\alpha_{\mathrm{dis}} \sum_{l=1}^{\infty} \int_{0}^{v_{F}^{2} \Lambda^{2}} d x \frac{\left(i \omega_{n}+\mu-\Sigma_{\mathrm{dis}}\right)}{\left(i \omega_{n}+\mu-\Sigma_{\mathrm{dis}}\right)^{2}-x} \cos \left(\frac{2 \pi l x}{\omega_{c}^{2}}\right) \\
= & \Sigma_{\mathrm{dis}}^{0}+\Sigma_{\mathrm{dis}}^{\mathrm{osc}} .
\end{aligned}
$$

Here we introduced a cutoff $\Lambda$, restricting the analysis to the regime where the dispersion is linear. The first term $\Sigma_{\text {dis }}^{0}$ is the $l=0$ term and corresponds to the standard expression of the SCBA in a system without magnetic field. The second part $\Sigma_{\text {dis }}^{\text {osc }}$ describes the oscillations of the self-energy due to the magnetic field. Performing the integration yields

$$
\Sigma_{\mathrm{dis}}^{0}=-\alpha_{\mathrm{dis}}\left(i \omega_{n}+\mu-\Sigma_{\mathrm{dis}}\right) \ln \frac{v_{F}^{2} \Lambda^{2}-\left(i \omega_{n}+\mu-\Sigma_{\mathrm{dis}}\right)^{2}}{-\left(i \omega_{n}+\mu-\Sigma_{\mathrm{dis}}\right)^{2}} .
$$


This expression can be solved self-consistently to leading order in $\alpha_{\text {dis }}$ and we obtain

$$
\Sigma_{\mathrm{dis}}^{0}=-\alpha_{\mathrm{dis}}\left(i \omega_{n}+\mu\right) \ln \frac{v_{F}^{2} \Lambda^{2}-\left(i \omega_{n}+\mu\right)^{2}}{-\left(i \omega_{n}+\mu\right)^{2}} .
$$

The oscillatory part, $\Sigma_{\text {dis }}^{\text {osc }}$, is treated by using the residue theorem,

$$
\begin{aligned}
\Sigma_{\mathrm{dis}}^{\mathrm{osc}}= & \alpha_{\mathrm{dis}}\left(i \omega_{n}+\mu-\Sigma_{\mathrm{dis}}\right) \sum_{l=1}^{\infty} \int_{0}^{\infty} d x \frac{e^{i\left(2 \pi l x / \omega_{c}^{2}\right)}+e^{-i\left(2 \pi l x / \omega_{c}^{2}\right)}}{\left(i \omega_{n}+\mu-\Sigma_{\mathrm{dis}}\right)^{2}-x} \\
= & \alpha_{\mathrm{dis}}\left(i \omega_{n}+\mu-\Sigma_{\mathrm{dis}}\right) 2 \pi i \Theta(a) \sum_{l=1}^{\infty} e^{-\left(2 \pi / \omega_{c}^{2}\right) \mid b l l}\left[e^{i\left(2 \pi / \omega_{c}^{2}\right) a l} \Theta(b)-e^{-i\left(2 \pi / \omega_{c}^{2}\right) a l} \Theta(-b)\right] \\
& +\alpha_{\mathrm{dis}}\left(i \omega_{n}+\mu-\Sigma_{\mathrm{dis}}\right) \sum_{l=1}^{\infty} \int_{0}^{\infty} d x\left(\frac{e^{-\left(2 \pi / \omega_{c}^{2}\right) x l}}{a+i(b-x)}-\frac{e^{-\left(2 \pi / \omega_{c}^{2}\right) x l}}{a+i(b+x)}\right)
\end{aligned}
$$

with $a=\left(\mu-\Sigma_{\text {dis }}^{\prime}\right)^{2}-\left(\omega_{n}-\Sigma_{\text {dis }}^{\prime \prime}\right)^{2}$ and $b=2\left(\omega_{n}-\Sigma_{\text {dis }}^{\prime \prime}\right)\left(\mu-\Sigma_{\text {dis }}^{\prime}\right)$. $\Sigma_{\text {dis }}^{\prime}$ is the real part of $\Sigma_{\text {dis }}$ and $\Sigma_{\text {dis }}^{\prime \prime}$ is its imaginary part. The first term stems from the residue. Whether the pole is located inside or outside the integration contour depends on the sign of $a$ and $b$. The second term stems from integration along the imaginary axis and is a nonoscillatory correction term. The integration contours used here are plotted in Fig. 2 . In the regime $T \gtrsim \omega_{c}$ both terms in $\Sigma_{\text {dis }}^{\text {osc }}$ are suppressed exponentially due to the factors $\exp \left(-\frac{2 \pi}{\omega_{c}^{2}}|b| l\right)$ and $\exp \left(-\frac{2 \pi}{\omega_{c}^{2}} x l\right)$, respectively. Thus, in this regime, $\Sigma_{\text {dis }}^{\text {osc }}$ can be neglected, and the self-energy can be well approximated as

$$
\Sigma_{\mathrm{dis}} \approx \Sigma_{\mathrm{dis}}^{0}
$$

\section{APPENDIX C: OSCILLATORY GRAND POTENTIAL WITH DISORDER}

In this section we will compute the grand potential for graphene with disorder, Eq. (17),

$$
\tilde{\Omega}=-\frac{T \omega_{c}^{4} L^{2}}{\pi^{2} v_{F}^{2}} \sum_{\omega_{n}} \sum_{l=1}^{\infty} \frac{1}{l} \int_{0}^{\infty} d x \frac{\sin (2 \pi l x)}{\left[i \omega_{n}+\mu+\Sigma_{\mathrm{dis}}\right]^{2}-\omega_{c}^{2} x} .
$$

The computation is analog to the one for the clean system which we perform in Appendix A. However, it is trickier to find the location of the pole, which is needed to evaluate this integral using the residue theorem. The pole is given by

$$
\begin{aligned}
x & =\frac{1}{\omega_{c}^{2}}\left[-i \omega_{n}+\mu+\Sigma_{\mathrm{dis}}\right]^{2} \\
& =\frac{1}{\omega_{c}^{2}}\left(i \omega_{n}-\mu\right)^{2}\left(1+\alpha_{\mathrm{dis}} \ln \left[\frac{\left(v_{F} \Lambda\right)^{2}}{-\left(i \omega_{n}-\mu\right)^{2}}\right]\right)^{2} .
\end{aligned}
$$

We expand the pole to linear order in $\alpha_{\text {dis }}$ as we are interested in a weak disorder potential.

$$
\omega_{c}^{2} x \approx\left(\mu^{2}-\omega_{n}^{2}\right)\left(1+\alpha_{\mathrm{dis}} \ln \left[\frac{v_{F} \Lambda}{r}\right]^{2}\right)-2 \omega_{n} \mu \alpha_{\mathrm{dis}}(\pi-2 \phi)-i\left\{2 \omega_{n} \mu\left(1+\alpha_{\mathrm{dis}} \ln \left[\frac{v_{F} \Lambda}{r}\right]^{2}\right)+\left(\mu^{2}-\omega_{n}^{2}\right) \alpha_{\mathrm{dis}}(\pi-2 \phi)\right\}
$$

with $r=\omega_{n}^{2}+\mu^{2}$ and $\phi=\arctan \left(\frac{\omega_{n}}{\mu}\right)$. The imaginary part is always smaller than zero. In order to find the zero crossing of the real part we use the ansatz $\mu=\omega_{n}+\delta \mu$ and expand the real part up to first order in $\delta \mu$. We find the zero crossing at $\mu=\omega_{n}\left(1+\alpha_{\text {dis }} \frac{\pi}{2}\right)$ such that only frequencies $\left|\omega_{n}\right|<\frac{|\mu|}{1+\alpha_{\text {dis }} \frac{\pi}{2}}$ contribute to the oscillations. We find

$$
\begin{aligned}
\tilde{\Omega}= & -\frac{T \omega_{c}^{4} L^{2}}{\pi^{2} v_{F}^{2}} \sum_{l=1}^{\infty} \frac{1}{l} \sum_{\omega_{n}>0} \int_{0}^{\infty} d x e^{-2 \pi l x}\left[\left(\mu+\Sigma_{\mathrm{dis}}^{\prime}\right)^{2}-\left(\omega_{n}-\Sigma_{\mathrm{dis}}^{\prime \prime}\right)^{2}\right] \\
& \times\left(\frac{1}{\left[\left(\mu+\Sigma_{\mathrm{dis}}^{\prime}\right)^{2}-\left(\omega_{n}-\Sigma_{\mathrm{dis}}^{\prime \prime}\right)^{2}\right]^{2}+\left[2\left(\mu+\Sigma_{\mathrm{dis}}^{\prime}\right)\left(\omega_{n}-\Sigma_{\mathrm{dis}}^{\prime \prime}\right)+\omega_{c}^{2} x\right]}\right. \\
& \left.+\frac{1}{\left[\left(\mu+\Sigma^{\prime}\right)^{2}-\left(\omega_{n}-\Sigma^{\prime \prime}\right)^{2}\right]^{2}+\left[2\left(\mu+\Sigma^{\prime}\right)\left(\omega_{n}-\Sigma^{\prime \prime}\right)-\omega_{c}^{2} x\right]}\right)
\end{aligned}
$$




$$
\begin{aligned}
& +\frac{2 T \omega_{c}^{2} L^{2}}{\pi v_{F}^{2}} \sum_{l=1}^{\infty} \frac{1}{l} \sum_{\omega_{n}>0}^{|\mu| /\left[1+\alpha_{\mathrm{dis}}(\pi / 2)\right]} e^{-\left(4 \pi l / \omega_{c}^{2}\right)\left[\left(\mu^{2}-\omega_{n}^{2}\right)\left(\pi \alpha-2 \phi \alpha_{\mathrm{dis}}\right)+|\mu| \omega_{n}\left(1+2 \alpha_{\mathrm{dis}} \Gamma\right)\right]} \\
& \times \cos \left(\frac{2 \pi l}{\omega_{c}^{2}}\left[\left(\mu^{2}-\omega_{n}^{2}\right)\left(1+2 \alpha_{\mathrm{dis}} \Gamma\right)+4 \alpha_{\mathrm{dis}}|\mu| \omega_{n}(2 \phi-\pi)\right]\right),
\end{aligned}
$$

where $\Sigma_{\text {dis }}^{\prime}$ is the real part of the self-energy and $\Sigma_{\text {dis }}^{\prime \prime}$ its imaginary part. $\Gamma$ is defined in (20). Here, again only the last term is oscillatory.

\section{APPENDIX D: THE INTERACTION INDUCED SELF-ENERGY}

In this section we will compute the interaction induced self-energy $\hat{\Sigma}_{e e}$ for graphene. The generic expression for the self-energy within RPA reads

$$
\hat{\Sigma}_{e e}\left(\mathbf{k}, i \omega_{n}\right)=-T \sum_{\omega_{n}^{\prime}} \int \frac{d^{2} k^{\prime}}{(2 \pi)^{2}} V\left(\mathbf{k}-\mathbf{k}^{\prime}, i \omega_{n}-i \omega_{n}^{\prime}\right) \hat{G}_{0}\left(\mathbf{k}^{\prime}, i \omega_{n}^{\prime}\right)
$$

The free-electron Green's function for graphene is given by

$$
\hat{G}_{0}\left(\mathbf{k}, i \omega_{n}\right)=\frac{-i \omega_{n} \mathbb{1}-v_{F} \mathbf{k} \hat{\boldsymbol{\sigma}}}{\omega_{n}^{2}+v_{F}^{2} k^{2}}
$$

and the Coulomb interaction $V\left(\mathbf{k}, i \omega_{n}\right)$ in the RPA approximation is given by

$$
V\left(\mathbf{k}, i \omega_{n}\right)=\frac{2 \pi \alpha v_{F}}{|\mathbf{k}|+\frac{2 \pi \alpha v_{F}}{4} \frac{k^{2}}{4 \sqrt{v_{F}^{2} k^{2}+\omega_{n}^{2}}},}
$$

with $\alpha=e^{2} /\left(\varepsilon v_{F}\right)$ ( $\varepsilon$ corresponds to the dielectric constant) being graphene's dimensionless fine-structure constant. In the following we will only work to two-loop accuracy and consequently expand the dressed Coulomb interaction to quadratic order yielding

$$
V\left(\mathbf{k}, i \omega_{n}\right)=\frac{2 \pi \alpha v_{F}}{k}-\frac{(2 \pi)^{2} \alpha^{2} v_{F}^{2}}{4 \sqrt{v_{F}^{2} k^{2}+\omega_{n}^{2}}}+O\left(\alpha^{3}\right) .
$$

We decompose the self-energy into a first-order and a second-order part according to

$$
\hat{\Sigma}_{e e}\left(\mathbf{k}, i \omega_{n}\right) \approx \hat{\Sigma}^{(1)}\left(\mathbf{k}, i \omega_{n}\right)+\hat{\Sigma}^{(2)}\left(\mathbf{k}, i \omega_{n}\right),
$$

where $\hat{\Sigma}^{(1)}\left(\mathbf{k}, i \omega_{n}\right)$ is linear in $\alpha$, while $\hat{\Sigma}^{(2)}\left(\mathbf{k}, i \omega_{n}\right)$ is quadratic in $\alpha$. They read

$$
\hat{\Sigma}^{(1)}\left(\mathbf{k}, i \omega_{n}\right)=2 \pi \alpha v_{F} T \sum_{\omega_{n}^{\prime}} \frac{d^{2} k^{\prime}}{(2 \pi)^{2}} \frac{1}{\left|\mathbf{k}-\mathbf{k}^{\prime}\right|} \frac{i \omega_{n}^{\prime} \mathbb{1}+v_{F} \mathbf{k}^{\prime} \hat{\boldsymbol{\sigma}}}{\omega_{n}^{\prime 2}+v_{F}^{2} k^{\prime 2}}
$$

and

$$
\hat{\Sigma}^{(2)}\left(\mathbf{k}, i \omega_{n}\right)=-\frac{(2 \pi)^{2} \alpha^{2} v_{F}^{2}}{4} T \sum_{\omega_{n}^{\prime}} \int \frac{d^{2} k^{\prime}}{(2 \pi)^{2}} \frac{1}{\sqrt{v_{F}^{2}\left(\mathbf{k}-\mathbf{k}^{\prime}\right)^{2}+\left(\omega_{n}-\omega_{n}^{\prime}\right)^{2}}} \frac{i \omega_{n}^{\prime} \mathbb{1}+v_{F} \mathbf{k}^{\prime} \hat{\boldsymbol{\sigma}}}{\omega_{n}^{\prime 2}+v_{F}^{2} k^{\prime 2}} .
$$

We see that the imaginary part is strictly diagonal, while the real part is off-diagonal. This motivates the ansatz given in Eq. (20) for the self-energy.

We start with the calculation of $\hat{\Sigma}^{(1)}$. From symmetry we observe that the diagonal part vanishes and only the off-diagonal part survives:

$$
\hat{\Sigma}^{(1)}\left(\mathbf{k}, i \omega_{n}\right)=2 \pi \alpha v_{F} T \sum_{\omega_{n}^{\prime}} \frac{d^{2} k^{\prime}}{(2 \pi)^{2}} \frac{1}{\left|\mathbf{k}-\mathbf{k}^{\prime}\right|} \frac{v_{F} \mathbf{k}^{\prime} \hat{\boldsymbol{\sigma}}}{\omega_{n}^{2}+v_{F}^{2} k^{2}}
$$

We apply an integral identity,

$$
\int_{-\infty}^{\infty} \frac{d x}{\pi} \frac{1}{a^{2}+x^{2}}=\frac{1}{a}
$$


and rescale $\mathbf{k}^{\prime}$ and $x$ with $v_{F}$, and obtain

$$
\hat{\Sigma}^{(1)}\left(\mathbf{k}, i \omega_{n}\right)=2 \pi \alpha T \sum_{\omega_{n}^{\prime}} \int \frac{d^{2} k^{\prime}}{(2 \pi)^{2}} \int \frac{d x}{\pi} \frac{1}{\left(\mathbf{k}^{\prime}-v_{F} \mathbf{k}\right)^{2}+x^{2}} \frac{\mathbf{k}^{\prime} \hat{\boldsymbol{\sigma}}}{\omega_{n}^{\prime 2}+k^{\prime} 2} .
$$

We use the Feynman parameter and write

$$
\begin{aligned}
\hat{\Sigma}^{(1)}\left(\mathbf{k}, i \omega_{n}\right) & =2 \pi \alpha T \sum_{\omega_{n}^{\prime}} \int \frac{d^{2} k^{\prime}}{(2 \pi)^{2}} \int \frac{d x}{\pi} \int_{0}^{1} d u \frac{\mathbf{k}^{\prime} \hat{\boldsymbol{\sigma}}}{\left[u\left(\mathbf{k}^{\prime}-v_{F} \mathbf{k}\right)^{2}+u x^{2}+(1-u)\left(\omega_{n}^{\prime 2}+k^{\prime 2}\right)\right]^{2}} \\
& =2 \pi \alpha T \sum_{\omega_{n}^{\prime}} \int \frac{d^{2} k^{\prime}}{(2 \pi)^{2}} \int \frac{d x}{\pi} \int_{0}^{1} d u \frac{\mathbf{k}^{\prime} \hat{\boldsymbol{\sigma}}}{\left[\left(\mathbf{k}^{\prime}-u v_{F} \mathbf{k}\right)^{2}+u x^{2}+u(1-u) v_{F}^{2} k^{2}+(1-u) \omega_{n}^{\prime 2}\right]^{2}} \\
& =2 \pi \alpha T \sum_{\omega_{n}^{\prime}} \int \frac{d^{2} k^{\prime}}{(2 \pi)^{2}} \int \frac{d x}{\pi} \int_{0}^{1} d u \frac{1}{\sqrt{u}} \frac{\left(\mathbf{k}^{\prime}+u v_{F} \mathbf{k}\right) \hat{\boldsymbol{\sigma}}}{\left[k^{\prime 2}+x^{2}+u(1-u) v_{F}^{2} k^{2}+(1-u) \omega_{n}^{\prime 2}\right]^{2}} .
\end{aligned}
$$

In the last step we shifted $\mathbf{k} \rightarrow \mathbf{k}^{\prime}+u v_{F} \mathbf{k}$ and rescaled $x$ and $\omega_{n}^{\prime}$. The $\mathbf{k}^{\prime}$ integral over the first summand vanishes as the integrand is odd; the second summand contributes

$$
\begin{aligned}
\hat{\Sigma}^{(1)}\left(\mathbf{k}, i \omega_{n}\right) & =-\frac{\alpha T v_{F} \mathbf{k} \hat{\boldsymbol{\sigma}}}{2} \sum_{\omega_{n}^{\prime}} \int \frac{d x}{\pi} \int_{0}^{1} d u \sqrt{u}\left(\frac{1}{\Lambda^{2}+x^{2}+u(1-u) v_{F}^{2} k^{2}+(1-u) \omega_{n}^{\prime 2}}-\frac{1}{x^{2}+u(1-u) v_{F}^{2} k^{2}+(1-u) \omega_{n}^{\prime 2}}\right) \\
& =-\frac{\alpha T v_{F} \mathbf{k} \hat{\boldsymbol{\sigma}}}{2} \sum_{\omega_{n}^{\prime}} \int_{0}^{1} d u \sqrt{u}\left(\frac{1}{\sqrt{\Lambda^{2}+u(1-u) v_{F}^{2} k^{2}+(1-u) \omega_{n}^{\prime 2}}}-\frac{1}{\sqrt{u(1-u) v_{F}^{2} k^{2}+(1-u) \omega_{n}^{\prime 2}}}\right) .
\end{aligned}
$$

The sum over $\omega_{n}^{\prime}$ can only be performed analytically in the limit $T \rightarrow 0$ when one can transform the sum into an integral,

$$
\begin{aligned}
\hat{\Sigma}^{(1)}\left(\mathbf{k}, i \omega_{n}\right) & =-\frac{\alpha v_{F} \mathbf{k} \hat{\boldsymbol{\sigma}}}{4 \pi} \int d \omega^{\prime} \int_{0}^{1} d u \sqrt{\frac{u}{1-u}}\left(\frac{1}{\sqrt{\Lambda^{2}+u(1-u) v_{F}^{2} k^{2}+\omega^{\prime 2}}}-\frac{1}{\sqrt{u(1-u) v_{F}^{2} k^{2}+\omega^{\prime 2}}}\right) \\
& =-\frac{\alpha v_{F} \mathbf{k} \hat{\boldsymbol{\sigma}}}{4 \pi} \int_{0}^{1} d u \sqrt{\frac{u}{1-u}} \ln \left(\frac{u(1-u) v_{F}^{2} k^{2}}{\Lambda^{2}+u(1-u) v_{F}^{2} k^{2}}\right) \\
& \approx-\frac{\alpha v_{F} \mathbf{k} \hat{\boldsymbol{\sigma}}}{4 \pi} \int_{0}^{1} d u \sqrt{\frac{u}{1-u}} \ln \left(\frac{u(1-u) v_{F}^{2} k^{2}}{\Lambda^{2}}\right) \\
& =\frac{\alpha v_{F} \mathbf{k} \hat{\boldsymbol{\sigma}}}{4} \ln \left(\frac{4 \Lambda}{v_{F} k}\right)
\end{aligned}
$$

Now we calculate $\hat{\Sigma}^{(2)}\left(\mathbf{k}, i \omega_{n}\right)$. We again use the integral identity Eq. (D9) and write

$$
\hat{\Sigma}^{(2)}\left(\mathbf{k}, i \omega_{n}\right)=-\frac{(2 \pi)^{2} \alpha^{2} v_{F}^{2}}{4} T \sum_{\omega_{n}^{\prime}} \int \frac{d^{2} k^{\prime}}{(2 \pi)^{2}} \int \frac{d x}{\pi} \frac{1}{x^{2}+v_{F}^{2}\left(\mathbf{k}-\mathbf{k}^{\prime}\right)^{2}+\left(\omega_{n}-\omega_{n}^{\prime}\right)^{2}} \frac{i \omega_{n}^{\prime} \mathbb{1}+v_{F} \mathbf{k}^{\prime} \hat{\boldsymbol{\sigma}}}{\omega_{n}^{\prime 2}+v_{F}^{2} k^{\prime 2}} .
$$

Using the standard Feynman parameter we can rewrite it as

$$
\hat{\Sigma}^{(2)}\left(\mathbf{k}, i \omega_{n}\right)=-\pi^{2} \alpha^{2} v_{F}^{2} T \sum_{\omega_{n}^{\prime}} \int \frac{d^{2} k^{\prime}}{(2 \pi)^{2}} \int \frac{d x}{\pi} \int_{0}^{1} d u \frac{i \omega_{n}^{\prime} \mathbb{1}+v_{F} \mathbf{k}^{\prime} \hat{\boldsymbol{\sigma}}}{u x^{2}+v_{F}^{2}\left(\mathbf{k}^{\prime}-u \mathbf{k}\right)^{2}+\left(\omega_{n}^{\prime}-u \omega_{n}\right)^{2}+u(1-u) \Omega^{2}}
$$

where $\Omega^{2}=v_{F}^{2} k^{2}+\omega_{n}^{2}$. In the following we analyze this expression in the zero-temperature limit. We note, however, that we have also analyzed the finite temperature behavior of this expression numerically, given in Eq. (33). In the limit $T \rightarrow 0$ we can rewrite the expression after an appropriate shift as

$$
\hat{\Sigma}^{(2)}\left(\mathbf{k}, i \omega_{n}\right)=-\pi^{2} \alpha^{2} v_{F}^{2} \int \frac{d \omega^{\prime}}{2 \pi} \int \frac{d^{2} k^{\prime}}{(2 \pi)^{2}} \int \frac{d x}{\pi} \int_{0}^{1} d u \frac{\left(i \omega^{\prime}+u i \omega_{n}\right) \mathbb{1}+v_{F}\left(\mathbf{k}^{\prime}+u \mathbf{k}\right) \hat{\boldsymbol{\sigma}}}{u x^{2}+v_{F}^{2} k^{\prime 2}+\omega^{\prime 2}+u(1-u) \Omega^{2}}
$$

For symmetry reasons odd integrals drop and we can simplify the expression to yield

$$
\hat{\Sigma}^{(2)}\left(\mathbf{k}, i \omega_{n}\right)=-\pi^{2} \alpha^{2}\left(i \omega_{n} \mathbb{1}+v_{F} \mathbf{k} \hat{\boldsymbol{\sigma}}\right) \int \frac{d \omega^{\prime}}{2 \pi} \int \frac{d^{2} k^{\prime}}{(2 \pi)^{2}} \int \frac{d x}{\pi} \int_{0}^{1} d u \frac{\sqrt{u}}{x^{2}+k^{\prime 2}+\omega^{\prime 2}+u(1-u) \Omega^{2}} .
$$


The integrals over $k^{\prime}$ with a cutoff $\Lambda$ and $\omega^{\prime}$ are elementary and we obtain

$$
\hat{\Sigma}^{(2)}\left(\mathbf{k}, i \omega_{n}\right)=-\frac{\alpha^{2}}{8}\left(i \omega_{n} \mathbb{1}+v_{F} \mathbf{k} \hat{\boldsymbol{\sigma}}\right) \int_{0}^{1} d u \sqrt{u} \int d x\left(\frac{1}{\sqrt{x^{2}+u(1-u) \Omega^{2}}}-\frac{1}{\sqrt{x^{2}+u(1-u) \Omega^{2}+\Lambda^{2}}}\right) .
$$

Integrating over $x$ leaves us with

$$
\hat{\Sigma}^{(2)}\left(\mathbf{k}, i \omega_{n}\right)=-\frac{\alpha^{2}}{8}\left(i \omega_{n} \mathbb{1}+v_{F} \mathbf{k} \hat{\boldsymbol{\sigma}}\right) \int_{0}^{1} d u \sqrt{u} \ln \frac{u(1-u) \Omega^{2}+\Lambda^{2}}{u(1-u) \Omega^{2}}
$$

which we can integrate to give

$$
\begin{aligned}
\hat{\Sigma}^{(2)}\left(\mathbf{k}, i \omega_{n}\right)= & -\frac{\alpha^{2}}{8}\left(i \omega_{n} \mathbb{1}+v_{F} \mathbf{k} \hat{\boldsymbol{\sigma}}\right) \int_{0}^{1} d u \sqrt{u} \ln \left(\frac{u(1-u) \Omega^{2}+\Lambda^{2}}{u(1-u) \Omega^{2}}\right) \\
= & \frac{\alpha^{2}}{24}\left(i \omega_{n} \mathbb{1}+v_{F} \mathbf{k} \hat{\boldsymbol{\sigma}}\right)\left\{\frac { \sqrt { 2 } } { \Omega ^ { 3 / 2 } } \left(\left(-\Omega+\sqrt{4 \Lambda^{2}+\Omega^{2}}\right)^{3 / 2} \arctan \left[\frac{\sqrt{2 \Omega}}{\sqrt{-\Omega+\sqrt{4 \Lambda^{2}+\Omega^{2}}}}\right]\right.\right. \\
& \left.\left.+\left(-\Omega-\sqrt{4 \Lambda^{2}+\Omega^{2}}\right)^{3 / 2} \arctan \left[\frac{\sqrt{2 \Omega}}{\sqrt{-\Omega-\sqrt{4 \Lambda^{2}+\Omega^{2}}}}\right]\right)+2 \ln \left[\frac{4 \Omega^{2}}{\Lambda^{2}}\right]\right\} .
\end{aligned}
$$

In the limit $\Omega^{2} \ll \Lambda^{2}$ this reduces to

$$
\hat{\Sigma}^{(2)}\left(\mathbf{k}, i \omega_{n}\right)=-\frac{\alpha^{2}}{2}\left(i \omega_{n} \mathbb{1}+v_{F} \mathbf{k} \hat{\boldsymbol{\sigma}}\right)\left[\frac{1}{6} \ln \left(\frac{\Lambda^{2} v_{F}^{2}}{k^{2} v_{F}^{2}+\omega_{n}^{2}}\right)-\frac{1}{3} \ln (2)+\frac{5}{9}\right] .
$$

[1] L. D. Landau, Sov. Phys. JETP 3, 920 (1957).

[2] V. N. Kotov, B. Uchoa, V. M. Pereira, F. Guinea, and A. H. Castro Neto, Rev. Mod. Phys. 84, 1067 (2012).

[3] J. González, F. Guinea, and M. A. H. Vozmediano, Phys. Rev. B 59, R2474(R) (1999).

[4] J. González, F. Guinea, and M. Vozmediano, Nucl. Phys. B 424, 595 (1994).

[5] D. C. Elias, R. V. Gorbachev, A. S. Mayorov, S. V. Morozov, A. A. Zhukov, P. Blake, L. A. Ponomarenko, I. V. Grigorieva, K. S. Novoselov, F. Guinea et al., Nat. Phys. 7, 701 (2011).

[6] G. L. Yu, R. Jalil, B. Belle, A. S. Mayorov, P. Blake, F. Schedin, S. V. Morozov, L. A. Ponomarenko, F. Chiappini, S. Wiedmann et al., Proc. Natl Acad. Sci. USA 110, 3282 (2013).

[7] D. V. Khveshchenko, Phys. Rev. Lett. 87, 246802 (2001).

[8] J. E. Drut and T. A. Lähde, Phys. Rev. Lett. 102, 026802 (2009).

[9] J. M. Luttinger and J. C. Ward, Phys. Rev. 118, 1417 (1960).
[10] J. E. Drut and T. A. Lähde, PoS (Lattice 2013) 498.

[11] I. M. Lifshitz and A. M. Kosevich, Sov. Phys. JETP 2, 636 (1955).

[12] D. Shoenberg, Magnetic Oscillations in Metals (Cambridge University Press, Cambridge, UK, 1984).

[13] Y. Adamov, I. V. Gornyi, and A. D. Mirlin, Phys. Rev. B 73, 045426 (2006).

[14] G. W. Martin, D. L. Maslov, and M. Y. Reizer, Phys. Rev. B 68, 241309 (2003).

[15] M. Fowler and R. E. Prange, Physics 1, 315 (1965).

[16] S. Engelsberg and G. Simpson, Phys. Rev. B 2, 1657 (1970).

[17] U. Briskot, I. A. Dmitriev, and A. D. Mirlin, Phys. Rev. B 87, 195432 (2013).

[18] V. P. Gusynin, V. A. Miransky, and I. A. Shovkovy, Phys. Rev. Lett. 73, 3499 (1994).

[19] D. T. Son, Phys. Rev. Lett. 98, 020604 (2007). 